\title{
On Spectral Theory of Sequences Vector Spaces in a Space without Scalar Product: Case of a Banach's Space G
}

\author{
S.V. Massamba \\ Département de MathématiqueetInformatique, Faculté des Sciences \\ Université Pédagogique Nationale (UPN) \\ Kinshasa, RD. Congo
}

\begin{abstract}
With the question of knowing if it is possible to study sequences in a nonseparable space or not provided with a scalar product, we propose a possible technique for an unspecified Banach's space $G$, with or without unit element, separable or not.
\end{abstract}

Keywords: Sequence, Convergent sequence, Vector space, Banach's space, Hilbert's space, Isomorphism.

\section{INTRODUCTION}

\subsection{Useful Vector Spaces of Sequences in G}

Let $\mathrm{G}$ be an arbitrary space of Banach, $G^{n}$ the vector space of sequences in G, i.e. $G^{n}=$ $\left\langle x=\left(x_{1}, x_{2}, \ldots, x_{i}, \ldots\right)\right.$ such that $x_{i} \in G$ for $\left.i=1,2,3, \ldots\right\rangle, G_{C}^{n}=\left\{x \in G^{n}: \exists x_{\alpha} \in\right.$ and $\lim _{i \rightarrow \infty} \| x_{i}-$ $x \alpha=0$ the vectorial subspace of convergent sequences in $\mathrm{G}$ and, finally, the vectorial subspace of $G_{C}^{n}$ denoted $G^{2}$ and defined as and by the following conditions:

$G^{2}=\left\{x=\left(x_{1}, x_{2}, \ldots, x_{i}, \ldots\right): x \in G_{C}^{n}\right.$ et $\left.\sum_{i=1}^{\infty}\left\|x_{i}\right\|^{2}<\infty\right\} ;$ it allows two vectorial subspaces denoted $G_{p}^{n}$ et $G^{P}$.

\subsection{Hilbert's spaces sequence}

Now, Let $\left(H_{i}\right)_{i \geq 1}$ be a sequence of unspecified spaces of Hilbert and $\mathrm{H}$ a set of sequences defined as follows: $\quad H=\left\{h=\left(h_{1}, h_{2}, \ldots, h_{i}, \ldots\right): h_{i} \in H_{i}\right.$ for $i=1,2,3, \ldots$ and $\left.\sum_{i=1}^{\infty}\left\|h_{i}\right\|^{2}<\infty\right\}$; it is known that $\mathrm{H}$ is a vector space such as, provided with the square form denoted and defined by $\alpha: H^{2} \rightarrow$ $\mathbb{R}: \alpha(h, g)=\sum_{i=1}^{\infty}\left(h_{i} \uparrow g_{i}\right)$ for all $h, g \in H$, it is a Hilbert's space called hilbertian sum of the sequence $\left(H_{i}\right)_{i \geq 1}[1]$.

\subsection{Towards the answer}

Let us return to vector spaces $G^{2}$ and $\mathrm{H}$, and consider the following maps: on the one hand $\gamma$ : $G^{2} \rightarrow H: x \rightarrow \gamma(x)=h \in H$ for all $x \in G^{2}$ and on the other hand $\omega:\left(G^{2}\right)^{2} \rightarrow H^{2}:(x, y) \rightarrow$ $\omega(x, y)=(g, h)$ for all $x, y \in G^{2}$ and $h, g \in H$; it is clear that $\gamma$ and $\omega$ are linear one-to-one maps, therefore isomorphisms $(i)$; now, Let $\left(G^{2}\right)^{2}: \varphi \rightarrow \longrightarrow \longrightarrow \mathbb{R}$ be a map ; it is easy and favorable to note that starting from three applications $\omega$, $\alpha e t \varphi$, one immediately obtains the diagram presented in section 2 . 
2. ANSWER

\subsection{Observing and exploiting the diagram}

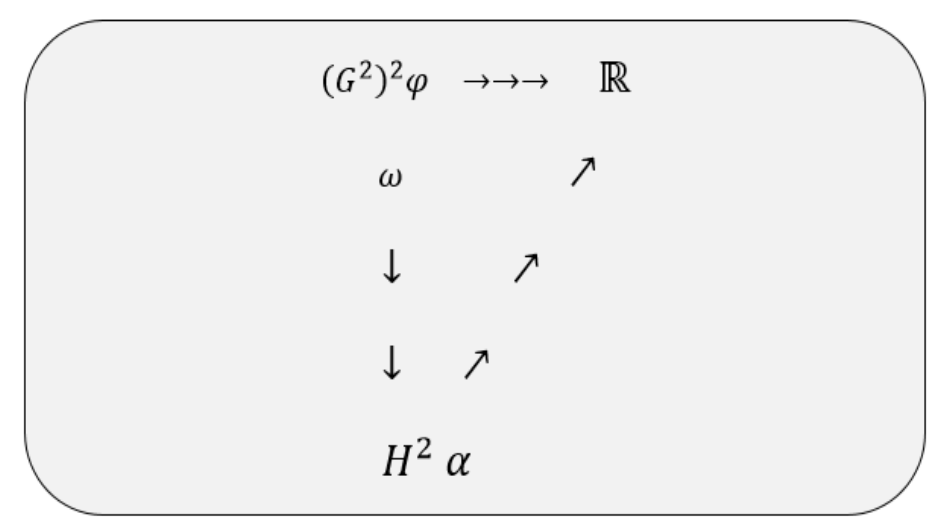

Figure 1. Commutative diagram

It is clear that $\varphi=\alpha o \omega$ such that, for all $x, y \in G^{2}$, one obtains following equalities :

$\varphi(x, y)=(\alpha \circ \omega)(x, y)=\alpha\{\omega((x, y))\}=\alpha(g, h)=\sum_{i=1}^{\infty}\left(g_{i} \uparrow h_{i}\right)$

or simply $\quad \varphi(x, y)=\sum_{i=1}^{\infty}\left(g_{i} \uparrow h_{i}\right)$

\subsection{Result}

As $\mathrm{H}$ and $G^{2}$ are isomorphic vector spaces $(i)$ and $\mathrm{H}$ is a Hilbert's space whose scalar product is $\alpha(h, g)=\sum_{i=1}^{\infty}\left(h_{i} \uparrow g_{i}\right)$ for all $h, g \in H$, it is easy to conclude that $G^{2}$ is a space of Hilbert whose scalar product is $\varphi(x, y)=\sum_{i=1}^{\infty}\left(x_{i} \uparrow y_{i}\right)$ for all $x, y \in G^{2}$; thus, one can easily establish the quadruple $G^{n}, G_{p}^{n}, G^{2}, G^{p}$ similar to the traditional quadruple $\mathbb{K}^{n}, \mathbb{K}_{p}^{n}, \ell^{2}, \ell_{p}$ attributed to Riesz.

\subsection{An illustration}

In particular, Let $\mathcal{B}(H)$ be a Banach's space; by representing a sequence in $\mathcal{B}(H)$ by $A=$ $\left(A_{1}, A_{2}, A_{3}, \ldots, A_{i}, ..\right)$ such that $A_{i} \in \mathcal{B}(H)$ for all $i=1,2,3, \ldots$ and one can consider the vector space of the convergent sequences in $\mathcal{B}(H)$, denoted and defined as follows:

$\mathcal{B}^{2}=\left\{A=\left(A_{1}, A_{2}, A_{3}, \ldots, A_{i}, ..\right)\right.$ such that $\left.\sum_{i=1}^{\infty}\left\|A_{i}\right\|^{2}<\infty\right\} ;$ it is provided with two vectorial subspaces denoted $\mathcal{B}_{p}^{n}$ and $\mathcal{B}^{p}$; it is easy to note that the vector space $\mathcal{B}^{2}$ is isomorphic to the vector space $G^{2}$ which is a Hilbert's space whose scalar product is denoted $\varphi(x, y)=\sum_{i=1}^{\infty}\left(x_{i} \uparrow y\right)$; it results from it that $\mathcal{B}^{2}$ is a space of Hilbert whose scalar product is written as $\delta(A, D)=\sum_{i=1}^{\infty}\left(A_{i} \uparrow\right.$ $D$ ffor all $A, D \in \mathcal{B} H$; thus, one can easily establish the quadruple $\mathcal{B} n, \mathcal{B} p n, \mathcal{B} 2, \mathcal{B} p$ similar to the traditional quadruple $\mathbb{K}^{n}, \mathbb{K}_{p}^{n}, \ell^{2}, \ell_{p}$ attributed to Riesz.

\section{ACKNOWLEDGEMENTS}

The author is grateful to Francis Mayala for typing the textof the present paper and to RuffinBenoîtNgoie for translating the original manuscript from French to English.

\section{REFERENCES}

[1] Dieudonné J., Eléments d'analyse moderne. Gautier Villars, Paris, 1974.

\section{AUTHOR's BIOGRAPHY}

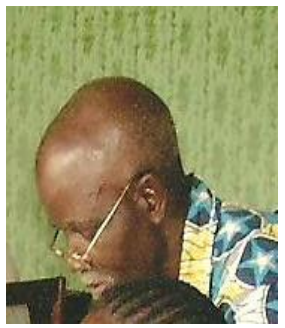

MasambaSalaVoka, is senior lecturer at UniversitéPédagogiqueNationale, Kinshasa (RDC). He teaches Infinitesimal Analysis, Functional Anlysis and General Topology. He is also a PhD. Student at the same University. 